\title{
Sonographic and pathological features of callosal hypoplasia in non-ketotic hyperglycinaemia
}

\author{
U K Wariyar, R J Welch, D W A Milligan, R H Perry
}

\begin{abstract}
A boy was born at 36 weeks' gestation weighing $2450 \mathrm{~g}$. Though his Apgar score was 9 at birth, by the age of $\mathbf{4 8}$ hours he required artificial ventilation. He was deeply unconscious with complete lack of muscle tone, and non-ketotic hyperglycinaemia associated with secondary hypoplasia of the corpus callosum was confirmed by biochemical tests. The cranial ultrasound scan features correlated well with the neuropathological findings and may be helpful in the early detection of this incurable condition.
\end{abstract}

Non-ketotic hyperglycinaemia is an autosomal recessive disorder with a distinctive neonatal presentation comprising hypotonia and coma in the absence of obvious metabolic disturbance. ${ }^{12}$ The primary pathology is dysmyelination of the brain and brain stem with associated microvacuolation. ${ }^{23}$ The most obvious macroscopic effect is secondary hypoplasia of the corpus callosum that causes the lateral ventricles to assume a 'bat's wing' appearance. ${ }^{2} 3$

Neonatal Unit,

Children's Department, Newcastle General Hospital,

Westgate Road,

Newcastle upon Tyne

NE4 6BE

U K Wariyar

R J Welch

D W A Milligan

Department of

Neuropathology,

Newcastle General

Hewcaste

R H Perry

Correspondence to: Dr Milligan.

Accepted 26 March 1990 ances of non-ketotic hyperglycinaemia have been described, ${ }^{4}$ there appears to have been no report to date of the cranial ultrasound features.

\section{Case report} gestation to a 21 year old single, primiparous mother with a stable non-consanguinous union. He required no resuscitation at birth (Apgar score 9 at one minute), but was described as 'floppy' by the nursing staff and needed tube feeding and mask resuscitation. At 38 hours of age he developed progressively severe apnoeic episodes, and by 48 hours required mechanical ventilation. He was transferred to this hospital and on examination was deeply unconscious (with no dysmorphic features), did not respond to painful stimuli, and made no spontaneous effort. There was complete lack of muscle tone but tendon reflexes were brisk. There were no abnormal movements and no signs of raised intracranial pressure. Mechanical ventilation was easy and blood gas measurements remained within normal limits in $25 \%$ oxygen. He had a grade $2 / 6$ systolic murmur over the left sternal edge. A clinical diagnosis of non-ketotic hyperglycinaemia was confirmed by the biochemical findings.

Plasma glycine concentration was 1676 $\mathrm{mmol} / \mathrm{l}$ (normal 200-400), urinary glycine 1100 $\mathrm{mmol} / \mathrm{mmol}$ creatinine (normal range 138-2100 $\mathrm{mmol} / \mathrm{mmol}$ creatinine), and cerebrospinal fluid (uncontaminated with blood) glycine was 551 $\mathrm{mmol} / \mathrm{l}$ (normal 10). Other amino acid concentrations, and the urinary organic acid profile, were normal. Plasma ammonia (as ammonium ion) was raised at $134 \mu \mathrm{mol} / \mathrm{l}$. After the diagnosis was confirmed active treatment was withdrawn and he died at 9 days of age.

A boy weighing $2450 \mathrm{~g}$ was born at 36 weeks'

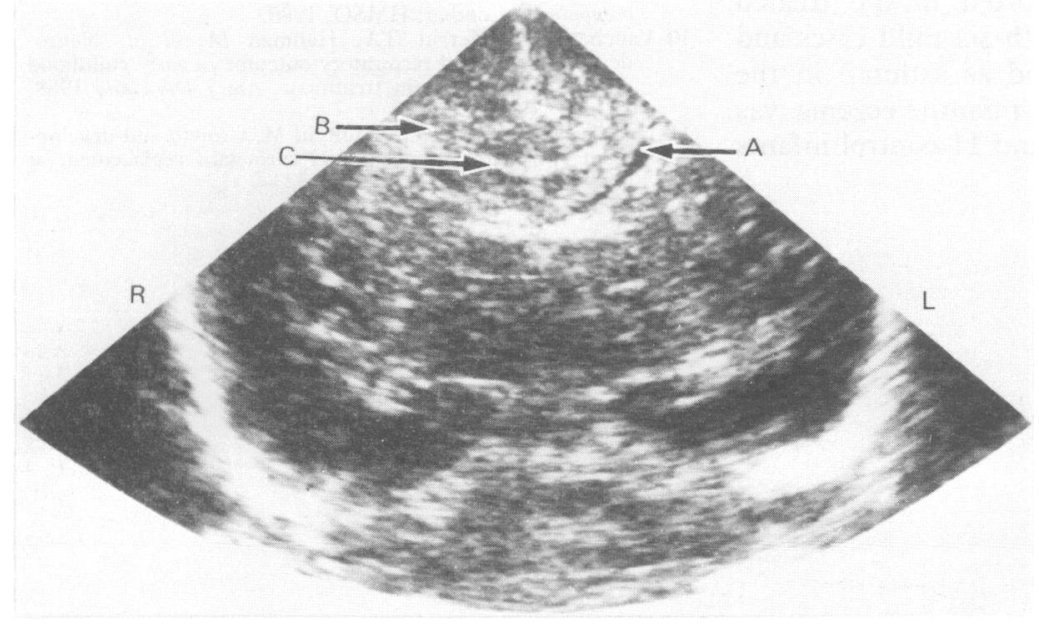

NEUROPATHOLOGICAL FINDINGS

After they had been fixed in formalin, coronal sections were examined; they showed a small (2-3 mm) but visible hypoplastic corpus callosum (fig 3 ) beneath a dorsally displaced cingulate gyrus. Histological examination of paraffin embedded sections showed a small band of central myelinated fibre bundles in the mid por-

Figure 1 Coronal ultrasound scan of the brain showing widely separated and horizontal lateral ventricles $(A)$ with areas of increased echogenicity containing small cysts in the periventricular white matter $(B) .(C=$ cingulate suclus, $L=$ left, $R=$ right. 


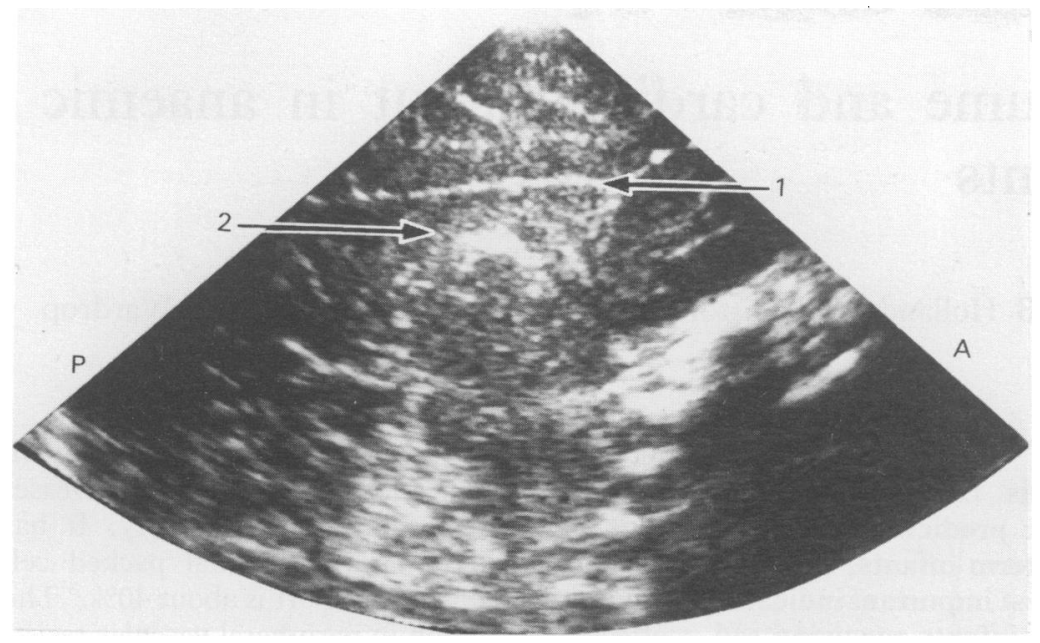

Figure 2 Midline sagittal ultrasound scan of the brain showing the cingulate sulcus (1) but no expected corpus callosum is visible in this area (2). $(A=$ anterior, $P=$ posterior.)

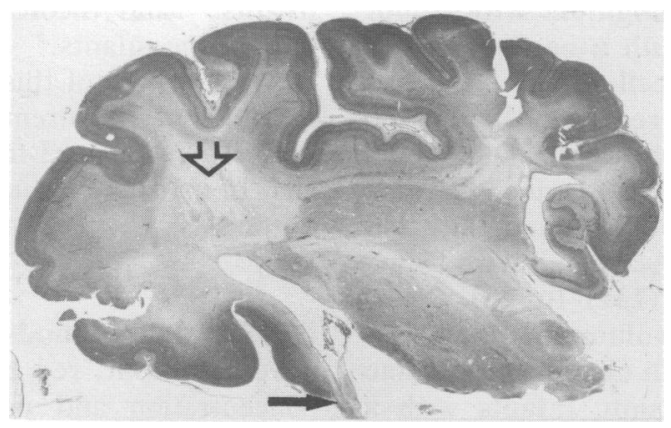

Figure 3 Right hemisphere coronal section (level of mamillary body) showing hypoplastic corpus callosum (solid arrow) and ventromedially displaced cingulate gyrus. Note that the fornix remains attached to the ventral (inferior) surface of the corpus callosum. Abnormalities of white matter are indicated (open arrow). (Formalin fixed, paraffin embedded $15 \mu$ cresyl violet stained section about one and a half times actual size.)

tion of the corpus callosum, which confirmed callosal hypoplasia. The central hemispheric white matter showed focal rarefaction and hypocellularity in the periventricular regions. In this region ependyma in the lateral ventricular angles (and beneath the corpus callosum) was present as an incomplete layer, and where it was absent the ventricular wall consisted solely of white matter and glial tissue. In these areas small ependymal rosettes were present suggesting that the ependymal layer had been disrupted or non-existent for some time before death.

\section{Discussion}

This patient had the clinical, biochemical, and pathological features of neonatal non-ketotic hyperglycinaemia. The neuropathological examination showed extensive dysmyelination of the cerebral white matter and hypoplasia of the corpus callosum, confirming the features seen on ultrasound scanning. Intracranial haemorrhage has previously been described, and it is interesting that the two cases described by Holmqvist and Polberger were infants born at full term.
Ultrasound comparison of corpus callosum hypoplasia and callosal agenesis

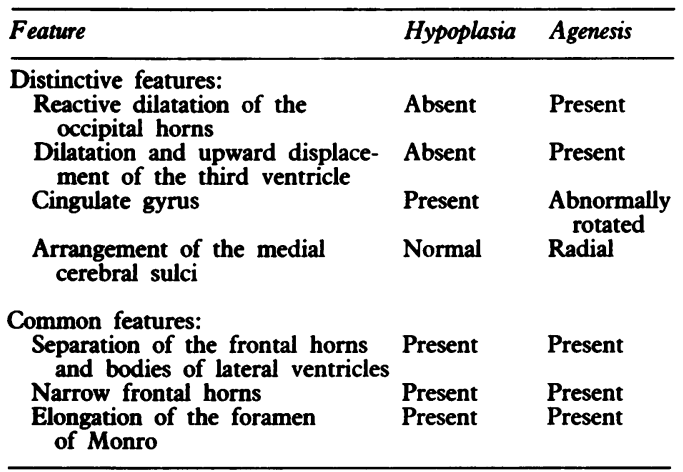

The ultrasonographic features of total and partial agenesis of the corpus callosum and its associated anomalies have been described in some detail, ${ }^{5-8}$ but although the possibility of secondary hypoplasia of the corpus callosum has been discussed, ${ }^{6}$ and cases have been described, ${ }^{9}$ the ultrasonographic features have not been delineated.

In the table the features of hypoplasia and agenesis of the corpus callosum are compared. It is apparent that disruption of a corpus callosum that is already partly formed has different effects on brain development from complete aplasia, and that these differences can be detected by ultrasonography.

Neonatal non-ketotic hyperglycinaemia is a devastating disorder for which no effective treatment is available. ${ }^{12}$ The presence of intracranial abnormalities at birth as seen in these and other patients suggests that any treatment will always have a limited success. ${ }^{23}$ In spite of this early recognition of the disorder is important so that appropriate management and parental counselling can be offered. Many of these babies will have cranial ultrasound scans during their intitial assessment. Recognition of the potential importance of callosal hypoplasia may give the clue to the real nature of the underlying disorder.

1 Holmqvist P, Polberger S. Neonatal non-ketotic hyperglycinaemia: diagnosis and management in two cases. Neuropediatrics 1985;16:191-3.

2 scher MS, Bergman I, Ahdab-Barmada M, Fria $T$ Neurophysiological and anatomical correlations in neonatal Neurophysiological and anatomical correlations in neonatal
non-ketotic hyperglycinaemia. Neuropediatrics 1986;17: non-ketotic

3 Shuman RM, Leech RW, Scott CR. The neuropathology of the non-ketotic and ketotic hyperglycinaemias: three cases. Neurology 1978;28:139-46.

4 Valavanis A, Schubiger O, Hayek J. Computed tomography in non-ketotic hyperglycinaemia. Computerised Tomography 1981;5:265-70.

5 Gebarski SS, Gebarski KS, Bowerman RA, Silver TM. Agenesis of the corpus callosum: sonographic features. Radio logy 1984;151:443-8.

6 Babcock DS. The normal and abnormal corpus callosum: sonographic findings. Radiology 1984;151:449-53.

7 Hernanz-Schulman M, Dohan FC Jr, Jones T, Cayea P Wallman J, Teele RL. Sonographic appearance of callosa Wenesis: correlation with radiographic and of callosal findings. AYNR 1985;6:361-8.

8 Atlas SW, Shkolnik A, Naidich TP. Sonographic recognition of agenesis of the corpus callosum. AfNR 1985;6:369-75.

9 Brun A, Probst F. The influence of associated cerebral lesions on the morphology of the acallosal brain. A pathological and $121-31$. 